Bio - grafía. Escritos sobre la Biología y su Enseñanza. ISSN 2027-1034

Edición Extraordinaria. p.p. 1386 - 1394

Memorias del IX Encuentro Nacional de Experiencias en Enseñanza de la Biología y la Educación Ambiental. IV Congreso Nacional de Investigación en Enseñanza de la Biología.

\title{
LA CONCEPCIÓN DE NATURALEZA SEGÚN LAS MIRAD AS DE COMENIUS Y VYGOTSKY: UNA AYUDA EN LA PRESERVACIÓN AMBIENTAL
}

\section{THE CONCEPTION OF NATURE ACCORDING TO THE LOOKS OF COMENIUS AND VYGOTSKY: AN AID TO ENVIRONMENTAL PRESERVATION}

\author{
Elizabeth Aparecida Assis Brandão Danhão ${ }^{1}$ \\ Rita de Cássia Frenedozo ${ }^{2}$ \\ Juliano Schimiguel ${ }^{3}$ \\ Ana Cristina Hurtado Coelho ${ }^{4}$ \\ Laura Marisa Carnielo Calejon
}

\section{RESUMEN}

La Educación Ambiental, así como otras áreas de enseñanza, han enfrentado el desafío de aproximar los contenidos ministrados en el aula con la vida del alumno. Para producir sentidos y significados, llevando la constitución de una conciencia de cuidado y preservación ambiental. Este artículo demuestra el ejercicio de estos principios en una estrategia de enseñanza destinada a diferentes edades y constituida por una visita de campo en un ambiente no formal cuyo objetivo es analizar las concepciones de naturaleza. Los resultados nos permitieron analizar las concepciones de naturaleza esencial propuesta por Comenius y la naturaleza elemental defendida por Vygotsky, de modo implícito y explícito, en la percepción de naturaleza del alumno y estimular su interrelación en la concientización de la preservación y conservación ambiental.

PALABRAS CLAVE: Interdisciplinaridad; Practica de Encino; Educación Ambiental.

1 Doutoranda em Ensino em Ciências e Matemática pela Universidade Cruzeiro do Sul. Bolsista CAPES. São Paulo-SP - Brasil. (profbethbrandao@gmail.com)

2 Professora Orientadora, Titular da Pós-Graduação em Mestrado e Doutorado em Ensino de Ciências e Matemática. Universidade Cruzeiro do Sul. São Paulo, Brasil. (rita.frenedozo@cruzeirodosul.edu.br)

3 3Professor Coorientador, Titular da Pós-Graduação em Mestrado e Doutorado em Ensino de Ciências e Matemática. Universidade Cruzeiro do Sul. São Paulo, Brasil. (schimiguel@gmail.com)

4 4Professora Coorientadora e Coordenadora da ESEC - Campus da Penha e Gambelas. Universidade de Algarve Faro, Portugal. (acoelho@ualg.pt)

${ }^{5}$ Professora titular da Pós graduação em Ensino de Ciências, Universidade Cruzeiro do Sul, São Paulo, SP; Icalejon@ig.com.br 
Bio - grafía. Escritos sobre la Biología y su Enseñanza. ISSN 2027-1034

Edición Extraordinaria. p.p. 1386 - 1394

Memorias del IX Encuentro Nacional de Experiencias en Enseñanza de la Biología y

la Educación Ambiental. IV Congreso Nacional de Investigación en Enseñanza de la Biología.

\begin{abstract}
Environmental Education, as well as other areas of education, has faced the challenge of bringing contents delivered in the classroom with the life of the student. In order to produce meanings and meanings, leading to the constitution of an awareness of environmental care and preservation. This article demonstrates the exercise of these principles in a teaching strategy destined to different age groups and constituted by a field visit in a no formal environment whose objective is to analyze the conceptions of nature. The results allowed us to analyze the conceptions of essential nature proposed by Comenius and the elemental nature defended by Vygotsky implicitly and explicitly in the student's perception of nature and to stimulate their interrelation in the awareness of environmental preservation and conservation.
\end{abstract}

KEY WORDS: Interdisciplinary; Practice Teaching; Environmental Education.

\title{
INTRODUCCIÓN
}

Las cuestiones ambientales hoy están en evidencia debido a la constante necesidad de preservación, equilibrio y de las amenazas sufridas por el planeta. La Educación Ambiental asume un importante papel interdisciplinario en la construcción de valores sociales, conocimientos, habilidades, actitudes y competencias dirigidas a la conservación del medio ambiente, que es un bien de uso común (Rivelli, 2005). Las visitas de campo, senderos ecológicos, actividades al aire libre, otras prácticas, dinámicas promueven una divulgación y una participación del alumno como componente del ambiente protagonista. Esta divulgación también despierta el interés de la sociedad por la conservación del medio ambiente (Rocha, 2002). Este trabajo propone la percepción de diferentes naturalezas existentes en una visita de campo o pista ecológica. La comprensión de las posibilidades de esta estrategia de enseñanza, constituidas a partir de la interacción del ser humano constituyente y activo en la naturaleza, se sustenta en pensamientos e ideas desarrolladas por Comenius y autores como Vygotsky. El desarrollo humano, las relaciones entre el aprendizaje y el desarrollo propuestas por Vygotsky, así como la comprensión de la naturaleza cultural de lo humano, permiten comprender la posibilidad de organizar la estrategia de campo en entornos no formales propuestos en este artículo. Estimulando as í la percepción de las diferentes naturalezas que tienden a estimular la concientización de la importancia y la preservación ambiental. Sin embargo, para comprender las diferentes naturalezas encontradas en una visita de campo, tenemos que partir de conceptos básicos sobre su significado. Para ello, analizamos los conceptos implícitos en teorías sobre la educación como la elaborada por Comenius y en las proposiciones de Vygotsky sobre la naturaleza humana y el papel de la educación en el desarrollo del sujeto. Así, las diferentes naturalezas que pueden ser rescatadas en una visita de campo, a través de una integración de los alumnos involucrados en un proceso de enseñanza aprendizaje interdisciplinario de educación ambiental.

\section{La epistemología pedagógica en la enseñanza de la naturaleza según Comenius}


Bio - grafía. Escritos sobre la Biología y su Enseñanza. ISSN 2027-1034

Edición Extraordinaria. p.p. 1386 - 1394

Memorias del IX Encuentro Nacional de Experiencias en Enseñanza de la Biología y la Educación Ambiental. IV Congreso Nacional de Investigación en Enseñanza de la Biología.

La definición de naturaleza es compleja y para intentar definirla partimos de la etimología de la palabra naturaleza: proveniente del latín Natura (natus/nascere) = nacer y urus/oritur = surgir, generar o la fuerza que genera. De esta definición podemos ampliar sus perspectivas filosóficas, o direccionarlas a una determinada ciencia específica. Mostrar las diferentes miradas que se tienen de la definición de naturaleza no es una tarea fácil. Es un tema que se extiende desde épocas remotas y que ha generado mucha discusión, polémica e incluso ideologías específicas para dominios individualistas, frenando luchas por poderes.

La pedagogía pasó por varias transformaciones y perfecciones. Vamos a enfatizar la influencia y la visión pedagógica de la naturaleza relatada por Juan Amós Comenius (1592-1670). Hemos elegido a Juan Amós Comenius por su visión humanista, por su necesidad constante de defender la interacción del individuo con el medio y con la sociedad y por su concepción de naturaleza esencial, pues para concientizar al ser humano de que es necesario cuidar, preservar, En el que vive, son necesarias acciones y actividades que hagan que esta visión humanista interactiva con la sociedad y el ambiente en que éste se inserta.

Juan Amós Comenius fue considerado el "padre" de la Didáctica Moderna y uno de los mayores educadores del siglo XVII. Con su teoría humanista y espiritualista logró desarrollar propuestas didácticas que tomaban en cuenta el desarrollo del niño, la construcción del conocimiento a través de experiencias, de observaciones y de una aplicación dialogada. "Él predicaba la necesidad de la interdisciplinaridad, de la afectividad del educador y de un ambiente escolar aireado, hermoso, con espacio libre y ecológico. Estelo también entre las acciones por el educador: la coerción de los propósitos educativos entre la familia y la escuela, el desarrollo del raciocinio y el espíritu y la formación del hombre religioso, social, político, racional, afectivo y moral ".

Él creía que la educación científica era fundamental para la perfección y para la salvación humana y que los sentidos eran necesarios para el conocimiento del medio externo e interno.

Básicamente Comenius presenta dos definiciones de naturaleza. Primero, la naturaleza en que se refiere al hombre, es su condición primera y fundamental que antecede a cualquier modificación [...]. En segundo lugar, Comenius comprende la naturaleza mientras la providencia universal de Dios [...] la bondad divina como élan vital que sostiene todas las cosas y seres que componen el mundo (Orbis) y con ello la naturaleza no se vincula exclusivamente al acto Creador, pero también al acto divino que sostiene todo el orden y armonía entre las cosas. La naturaleza, en esa concepción, es más que la creación divina: es la propia fuerza (divina) que sostiene las cosas en su existencia "(Nalli, 2003, p.77).

En la concepción de Comenius la naturaleza es una parte de Dios que el hombre es integrante y si él conoce el ambiente que el rodeo estaría conociendo a Dios ya sí mismo. Esta relación del hombre, con Dios y con el ambiente en que se encuentra nos da una visión de interdependencia y de un vínculo formador único, donde, si Dios es la fuerza generadora, que constituye la naturaleza y el ser humano, ambos son parte 
Bio - grafía. Escritos sobre la Biología y su Enseñanza. ISSN 2027-1034

Edición Extraordinaria. p.p. 1386 - 1394

Memorias del IX Encuentro Nacional de Experiencias en Enseñanza de la Biología y

la Educación Ambiental. IV Congreso Nacional de Investigación en Enseñanza de la Biología.

Integrante y dependiente del medio donde están insertados. Esta interacción es fundamental para la percepción de su papel en el medio biótico y abiótico. A partir de la visión de Comenius el ser humano es parte constituyente del ambiente, que está formado por una fuerza mayor y que se relaciona e interprende unos de otros y del ambiente que lo rodea.

\section{Teorías de Aprendizaje en la concepción de "naturaleza" según Vygotsky}

En la perspectiva presentada anteriormente se destaca una visión humanista y el ser como parte constitutiva del ambiente. La proposición de la naturaleza cultural del hombre resulta en la comprensión de que el ambiente en que el hombre vive está constituido por el propio hombre en la forma de diferentes contenidos de cultura. Así, Vygotsky afirma que al construir la cultura, el hombre se construye a sí mismo, construye aún el ambiente en que va a vivir y la calidad de las relaciones interpersonal que establece. Algunos datos sobre Vygotsky permiten comprender de manera más adecuada sus proposiciones y su contribución a la organización de escenarios de enseñanza.

Él nació en Orsha (Bielorrusia) el 17 de noviembre de 1896 en una familia judía. Se formó en Derecho y vivió en el auge de la Revolución Rusa. Se inició la carrera de profesor de Literatura y Psicología en Gomel y colaboró con editores. En 1919 contrajo tuberculosis, luego de haberse casado, teniendo dos hijas. Fue escritor, realizó conferencias, estudios y obras dirigidas a la psicología. En 1934 muere a los 37 años. Muchas obras de Vygotsky fueron publicadas y traducidas en el mundo después de su muerte. Vygotsky hace un análisis crítico de la psicología existente a principios del siglo XX, demostrando la necesidad de superar las dicotomías y el reduccionismo existente en las teorías de la época. También sugiere la importancia de la educación como fuerza transformadora de la sociedad y del desarrollo de las funciones psíquicas superiores del sujeto, incluyendo la conciencia y el dominio de la propia conducta. Esta nueva psicología que Vygotsky promulgaba sólo podía aparecer en una nueva sociedad cuando el ser humano pudiera mejorarse a sí mismo ya los resultados de su propia acción. Es necesario para comprender esta proposición considerar que diferentes del animal, el ser humano tiene una historia cultural que impacta su historia biológica, dando a ésta una nueva dirección. El ser humano es producto de un proceso histórico y de las transformaciones que él mismo produjo en la naturaleza y el ambiente en que vive, el pensamiento de Vygotsky fue interpretado de diferentes maneras, habiendo a menudo extirpada su base epistemológica, pero esta discusión sobrepasa límites De este artículo.

Para Vygotsky, los elementos mediadores en la relación entre el hombre y el mundo están constituidos en las relaciones entre los hombres ... Estos elementos están constituidos por instrumentos, signos y todos los elementos de la naturaleza humana irrigados de significado cultural, constituidos a través de las relaciones entre los hombres seres humanos., Que describe que la naturaleza y sus acciones colectivas influyen en la formación del ciudadano e interfieren en el proceso de enseñanza aprendizaje, tanto en la visión social, cultural y ambiental.

Buscamos describir la naturaleza elemental como la naturaleza física, biológica y química presente en el medio ambiente. La naturaleza que es fundamental para la 
Bio - grafía. Escritos sobre la Biología y su Enseñanza. ISSN 2027-1034

Edición Extraordinaria. p.p. 1386 - 1394

Memorias del IX Encuentro Nacional de Experiencias en Enseñanza de la Biología y

la Educación Ambiental. IV Congreso Nacional de Investigación en Enseñanza de la

Biología.

supervivencia de los seres humanos y que se relacionan en equilibrio, pero que pueden sufrir la interferencia irregular o inconsecuente y volverse desequilibrada. Es importante que el alumno perciba que esta naturaleza ha sido modificada por el hombre a lo largo de la historia de la propia humanidad y que si los cambios por un lado traen cierto confort y comodidad, por otro causan transformaciones de la naturaleza considerada Elemental. Los alumnos constituyen sus memorias a través de pensamientos e intercambios de experiencias y diálogos. Esta formación psíquica es voluntaria y se construye a lo largo de la vida. "El significado de todo está en las personas y en las cosas, pero él no puede estar en las cosas si no está en las personas, de ahí el papel de las emociones" (Vygotsky, 1994). Según Fariñas (2010, p.60) el estilo de vida convertido en unidad de análisis puede expresar dimensiones, convirtiéndose asumen una vía para el estudio del desarrollo de la personalidad y de los conceptos.

Es en esta teoría de Vygotsky que nos apoyamos para defender que las visitas de campo son importantes herramientas de aprendizaje, pues relacionan lo elemental con el intercambio de experiencias y la formación psíquica y emocional del alumno con la naturaleza biótica y abiótica. Esta interiorización proporciona subsidios para que el individuo encuentre la naturaleza esencial, formadora de lo social, cultural y religioso de cada uno.

Las emociones son significativas y no son dualistas en el momento de la visita de campo, pues van a estimular la interrelación del individuo con el medio ambiente en que está visitando y va a ilustrar con la práctica la aplicación teórica interdisciplinaria desarrollada en la actividad. Según él, las emociones influencian en la razón y cuando un alumno, por ejemplo, visualiza de forma natural un animal o una especie diferenciada en una práctica ambiental, acciona el proceso llamado de síntesis dialéctica, que se encuentra en la necesidad de lo nuevo o inexistente.

\section{La Educación Ambiental en una perspectiva interdisciplinaria}

La educación ambiental surgió de la necesidad de concientizar a los seres humanos de la importancia del conocimiento, de la preservación de la naturaleza, de recursos naturales y de soluciones a problemas ambientales. Este término surgió aproximadamente en 1972, en la primera Conferencia Mundial del Medio Ambiente Humano en Estocolmo (Suecia) realizada por la ONU. En 1975, la UNESCO (Organización de las Naciones Unidas para la Educación, la Ciencia y la Cultura) asumió la responsabilidad de organizar y debatir la Educación Ambiental de forma regional e internacional1. A partir de ahí la educación ambiental se ha convertido en una herramienta para auxiliar en las técnicas de aprendizaje.

Según Sauvé (2005), se trata de una dimensión esencial de la educación fundamental que se refiere a una esfera de interacciones que está en la base del desarrollo personal y social: la de la unción con el medio en que vivimos, con esa "casa de vida "Compartida. Más que una educación "respecto al, para él, por el o por el medio ambiente, el objeto de la educación es, fundamentalmente, nuestra relación con el medio ambiente. 
Bio - grafía. Escritos sobre la Biología y su Enseñanza. ISSN 2027-1034

Edición Extraordinaria. p.p. 1386 - 1394

Memorias del IX Encuentro Nacional de Experiencias en Enseñanza de la Biología y la Educación Ambiental. IV Congreso Nacional de Investigación en Enseñanza de la Biología.

La Educación Ambiental es muy compleja, pues engloba diferentes aspectos. Se trata de un tema interdisciplinario y esencial para la supervivencia del planeta. Entre ellos podemos relacionar: el medio ambiente de acuerdo con el enfoque que se pretende dirigir, por ejemplo: naturaleza para las relaciones interpersonal de los seres humanos y de la naturaleza sean ellas de preservación o de relajación, pero unidas; Para el medio ambiente como recurso de materia y energía; Como problema, cuando existen necesidades de resoluciones; El medio ambiente del sistema, para ser utilizado en la comprensión o decisión; Como lugar donde se vive; Como biosfera; Como proyecto comunitario, entre otros.

Mostrar las diferentes miradas que se tienen en la definición de naturaleza no es una tarea fácil, aún más cuando intentamos utilizar esa definición en las aplicaciones Educación Ambiental. Son temas complejos y que presentan diferentes vertientes, son oriundos de épocas distantes en que muchos conceptos se distinguían y al mismo tiempo en que individualmente podían ser utilizados para fortalecer una teoría o una línea de raciocinio. Es en contacto con la "naturaleza" que el individuo puede observar, rescatar valores, involucrarse y regresar a su íntimo biológico. Dependiendo del enfoque disciplinario que se pretende trabajar, las visitas de campo son herramientas importantes para la fijación, concientización y aprendizaje del individuo.

\section{METODOLOGÍA}

La propuesta de actividades en ambiente no formal despierta en el alumno la curiosidad y la expectativa de la ilustración de la teoría aplicada en el aula, estimulando así la búsqueda de la naturaleza esencial y elemental individual y colectiva. Esta salida de campo ocurrió con 35 alumnos de la enseñanza fundamental II, en el Parque de Vila Formosa cerca de una escuela estatal de Sorocaba interior de São Paulo / SP - Brasil. Los alumnos presentaban edades variadas entre 11 y 15 años y estaban participando en una disciplina electiva orientada al estudio de la Educación Ambiental y Biológica.

Los alumnos fueron acompañados por dos profesores responsables y recibieron a la comanda de observar todos los elementos de la naturaleza desde la salida de la escuela hasta el regreso. En un determinado momento los alumnos fueron orientados a sentarse cerca de un árbol y reflejar los conceptos de naturaleza. Se agruparon y organizaron en una gran rueda e introducimos las siguientes preguntas: ¿Cuál es el concepto de naturaleza? ¿Cuál es el significado de naturaleza esencial y elemental? ¿Cuál sería el papel de cada ser vivo de forma individual y colectiva? Como cada uno de los alumnos participan en ese contexto y de qué forma?

Cada alumno se manifestó e iniciando así un debate sobre los conceptos. Las concepciones iniciales fueron registradas por un profesor, mientras que el otro aplicaba los cuestionamientos y provocaciones para un debate. Cuando regresaron al aula se solicitaron los registros, en el cuaderno escolar, de la experiencia y de las reflexiones en el Parque ubicado en el entorno escolar. 
Bio - grafía. Escritos sobre la Biología y su Enseñanza. ISSN 2027-1034

Edición Extraordinaria. p.p. 1386 - 1394

Memorias del IX Encuentro Nacional de Experiencias en Enseñanza de la Biología y la Educación Ambiental. IV Congreso Nacional de Investigación en Enseñanza de la Biología.

\section{LOS RESULTADOS}

De acuerdo con los cuestionamientos realizados vamos a destacar algunos testimonios en la tabla abajo. Indicamos a los alumnos como A1, A2, A3 y así sucesivamente.

\begin{tabular}{|c|c|}
\hline Pregunta & stas \\
\hline $\begin{array}{l}\text { ¿Cuál es el concepto de } \\
\text { naturaleza? }\end{array}$ & $\begin{array}{l}\text { A1- "Es el conjunto de vidas" } \\
\text { A2 - "Donde quedan todos los seres vivos" } \\
\text { A3 - "Es la vida!". } \\
\text { A4 - "donde vivimos y seres vivos" }\end{array}$ \\
\hline $\begin{array}{l}\text { ¿Cuál es el significado de } \\
\text { naturaleza esencial y } \\
\text { elemental? }\end{array}$ & $\begin{array}{l}\text { A1 - "Esencial es todo lo que va a satisfacer nuestras } \\
\text { necesidades y elemental es lo mismo". } \\
\text { A2 - "Esencial es cuando usted necesita y necesita cierta cosa de } \\
\text { la naturaleza y Elemental sería agua, tierra, etc.". } \\
\text { A3 - "Esencial serían las plantas para nuestra supervivencia y la } \\
\text { elemental los elementos que son necesarios para el día a día" } \\
\text { A4 - "Esencial es todo lo que el ser humano necesita y elemental" }\end{array}$ \\
\hline $\begin{array}{l}\text { ¿Cuál sería el papel de } \\
\text { cada ser vivo de forma } \\
\text { individual y colectiva? }\end{array}$ & $\begin{array}{l}\text { A1 - Individual es desarrollarse y colectiva es ayudar a otros } \\
\text { seres a desarrollarse " } \\
\text { A2 - "Individualmente es vivir, respirar y colectivamente cada uno } \\
\text { tiene que tener una responsabilidad sobre el otro". } \\
\text { A3 - de forma individual es cumplir el ciclo de vida y en el grupo } \\
\text { es lo mismo. } \\
\text { A4 - "Individual es trabajar y colectivo ayudar al prójimo". }\end{array}$ \\
\hline $\begin{array}{l}\text { - ¿Cómo participan cada } \\
\text { uno de los alumnos en } \\
\text { ese contexto y de qué } \\
\text { forma? }\end{array}$ & $\begin{array}{l}\text { A1 - "Me adaptando las cosas y en el colectivo debo contribuir a } \\
\text { la adaptación de los demás". } \\
\text { A2 - "me desarrollando en el cuerpo y en la cabeza" } \\
\text { A3 - "Evolucionando y ayudando a otros alrededor" } \\
\text { A4 - "Observando el ambiente, participando de él y en el colectivo } \\
\text { tengo que actuar". }\end{array}$ \\
\hline
\end{tabular}

Tabla 1: Testimonios de los alumnos sobre los conceptos de naturaleza.

Según los relatos provenientes de la visita de campo, en el Parque de la Formosa, percibimos que el concepto amplio de naturaleza no está bien definido para los alumnos y que ellos reconocen su importancia y su papel en la naturaleza, como 
Bio - grafía. Escritos sobre la Biología y su Enseñanza. ISSN 2027-1034

Edición Extraordinaria. p.p. 1386 - 1394

Memorias del IX Encuentro Nacional de Experiencias en Enseñanza de la Biología y

la Educación Ambiental. IV Congreso Nacional de Investigación en Enseñanza de la Biología.

parte integrante y fundamental. Se percibe también que la visión de individualidad y colectivo se confunde, pero también se destacan de forma importante.

Siendo así, en esa actividad, tenemos la unión de la naturaleza esencial, formadora y constituyente del individuo siendo accionada para observar la naturaleza elemental ilustrada en las visitas de campo. Cuanto más experiencias el alumno agrega, pero él tendrá un aumento de sus interrelaciones sociales, de forma compleja y racional.

En esta visita de campo estimulamos la observación y podemos enfatizar la interacción del ser humano con la naturaleza, aunque desconocida conceptualmente, es reconocida instintivamente a través de los sentidos y la observación. Siendo así podemos dialogar con las colocaciones de Comenius, en el sentido de, aunque instintivamente, el ser humano se relaciona e interprenden unos de otros y del ambiente en que están insertados. Esta búsqueda por la naturaleza esencial hace perceptible la constitución y dependencia del hombre en el medio, lo que auxilia en la concientización de la preservación y conservación ambiental.

En cuanto a la naturaleza elemental propuesta por Vygotsky, queda visible en los testimonios de los alumnos que relacionan los factores químicos, físicos y biológicos observados y percibidos en la salida de campo en el Parque de la Formosa, a través de su percepción de su interdependencia y su influencia en los cuidados con la preservación Ambiental para la propia supervivencia. Otro punto es la interacción social que tenemos con los alumnos en esta visita de campo al Parque de la Formosa, pues cada ser presenta conocimientos previos que comparte y auxilia en la formación del conocimiento colectivo y de la interacción con la naturaleza. Este comportamiento social dialoga con las propuestas de Vygotsky. En la rueda de conversación los alumnos tuvieron entonces el contacto con la naturaleza exterior (elemental), el contacto con la naturaleza interior (esencial) y con el grupo social que acompaña la actividad. Estas evidencias se plantearon en las reflexiones provocadas por los profesores y en los registros finales de los alumnos.

\section{CONSIDERACIONES FINALES}

Buscamos, con esa actividad, elucidar el descubrimiento del alumno en su interrelación y su interdependencia de la naturaleza y de la sociedad, a través del reconocimiento de la naturaleza esencial (Comenius) y de la elemental (Vygotsky). Muchas son las explicaciones y teorías sobre naturaleza, pero cabe dejar claro que en nuestra concepción, la naturaleza esencial está presente y activa en el individuo, así como la naturaleza elemental, haciéndose complejo y al mismo tiempo único o simple, pues existen muchas naturalezas En el ser humano y todas ellas son constituyentes y formadoras esenciales de una naturaleza generadora, donde están interconectadas directa e indirectamente, por el ser humano. Es en la complejidad de esas diferentes naturalezas que buscamos, la reflexión, la conservación, la preservación, la recuperación y la concientización ambiental en las visitas de campo. 
Bio - grafía. Escritos sobre la Biología y su Enseñanza. ISSN 2027-1034

Edición Extraordinaria. p.p. 1386 - 1394

Memorias del IX Encuentro Nacional de Experiencias en Enseñanza de la Biología y la Educación Ambiental. IV Congreso Nacional de Investigación en Enseñanza de la Biología.

\section{REFERENCIAS}

Nalli, M. A. G. (2003). Consideraciones sobre el concepto de "naturaleza" en Comenius. Semina: Ciencias Sociales y Humanas: 75-86.

Rivelli, E. A. (2005). Evolución de la Legislación Ambiental en Brasil: Políticas de Medio Ambiente, Educación Ambiental y Desarrollo Urbano in PHILIPPI, Jr.; PELICIONI, MCF Educación Ambiental y Sostenibilidad. Barueri, SP (2005).

G., C. (2002). Gestión ambiental en municipios: riesgos e impactos ambientales. Curso de especialización en Gestión Ambiental en Municipios. Apostila. UFJF.

Sauvé, L. (1997). Educación ambiental y desarrollo sostenible: un análisis complejo. Revista de Educación Pública 6.10: 72-103.

Sauvé, L. (2005). Educación Ambiental: posibilidades y limitaciones. Educación e Investigación, São Paulo: 317-322.

Vygotsky, L.S. (1982): Obras T. II. ED. Pedagogika, Moskba.

(1994): El problema Del entorno. En: van der Veer, R. Valsiner, J. (Eds): The Vygotski reader. Oxford: Blackwell 